\title{
Quadrant analysis of turbulent pollution flux above the modelled street intersection
}

\author{
L. Kukačka ${ }^{1,2, a}$, Š. Nosek ${ }^{2}$, R. Kellnerová ${ }^{1,2}$, K. Jurčáková ${ }^{2}$, and Z. Jaňour ${ }^{2}$ \\ 1 Charles University in Prague, Faculty of Mathematics and Physics, The Department of Meteorology and Environment \\ Protection, Czech Republic \\ 2 Institute of Thermomechanics Academy of Sciences of the Czech Republic, v.v.i, Dolejškova 1402/5, Prague 182 00, \\ Czech Republic
}

\begin{abstract}
The objective of this experimental study is to determine processes of a vertical turbulent pollution transport above the $\mathrm{X}$-shaped street intersection in an idealised symmetric urban area for several approach flow directions. An experimental set-up for simultaneous measurement of the flow velocity and the tracer gas concentration in a high temporal resolution is assembled. Vertical turbulent scalar fluxes are computed from the measured data in a horizontal plane above the street intersection. The quadrant analysis was applied to the vertical turbulent pollution fluxes data. Events with dominant contribution to vertical turbulent pollution flux were detected. The mean duration, repetition frequency and the duration percentage were computed for these events. A strong influence of the approach flow direction on the the type of dominant events and their characteristics was resolved.
\end{abstract}

\section{Introduction}

Dispersion of air pollution within urban areas is an important aspect of the environment quality for a significant part of the population. Traffic in street canyons is often a dominant source of pollutants in large cities [1]. Improvement of air quality in urban areas is necessary to avoid risk for human health [2]. We focused on vertical turbulent pollution transport in a complex and highly three-dimensional flow and concentration fields above the idealised street intersection. This study relates to former published work [3].

Street intersections are very important in the redistribution of pollutants between streets and in the air exchanges between streets and flow above the canopy layer. Characteristics of the transport pollution within the street intersection can be found in recent works [4-6]. Understanding processes of pollution transport in complex urban areas is important for estimation of ventilation intensity in the polluted street canyons, for finding suitable configuration of built-up areas and for developing local scale dispersion models.

The quadrant analysis is usually the first step to investigate the turbulent processes in strongly turbulent flow. It is usually applied to the turbulent momentum flux [7,8]. Using this analysis, the prevailing events in the flow can be detected. There have been only several studies using quadrant analysis for turbulent scalar flux investigation, but only in the flow above relatively homogeneous surface, e.g. $[9,10]$. The quadrant analysis is applied newly to the turbulent scalar flux in highly turbulent a tree dimensional flow in this work.

\section{Experimental set-up}

\subsection{Wind tunnel}

The experiment was conducted in the open low-speed wind tunnel of Institute of Thermomechanics Academy of Sciences of the Czech Republic in Nový Knín. The crossdimension of the tunnel test section was $1.5 \times 1.5 \mathrm{~m}$, the length of the test section was $2 \mathrm{~m}$. The scheme of the tunnel is depicted in figure 1.

Fully turbulent boundary layer was developed by the $20.5 \mathrm{~m}$ long development section of the tunnel. This section was equipped by turbulent generators at the beginning and covered by $50 \mathrm{~mm}$ and $100 \mathrm{~mm}$ high roughness elements on the floor, see the photo in figure 2.

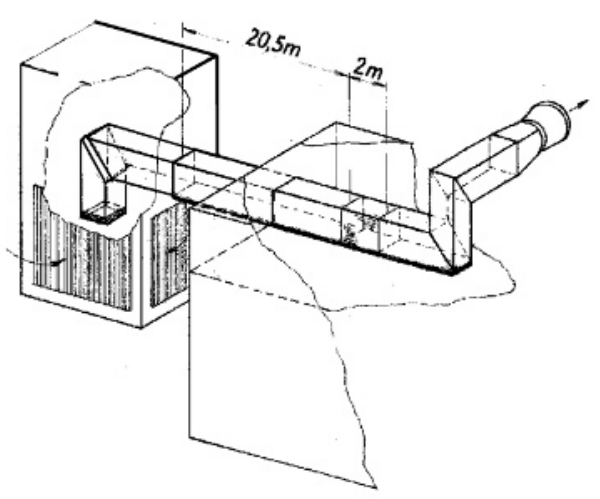

Fig. 1. The scheme of the open low-speed wind tunnel.

\footnotetext{
${ }^{a}$ e-mail: kukacka@it.cas.cz
} 


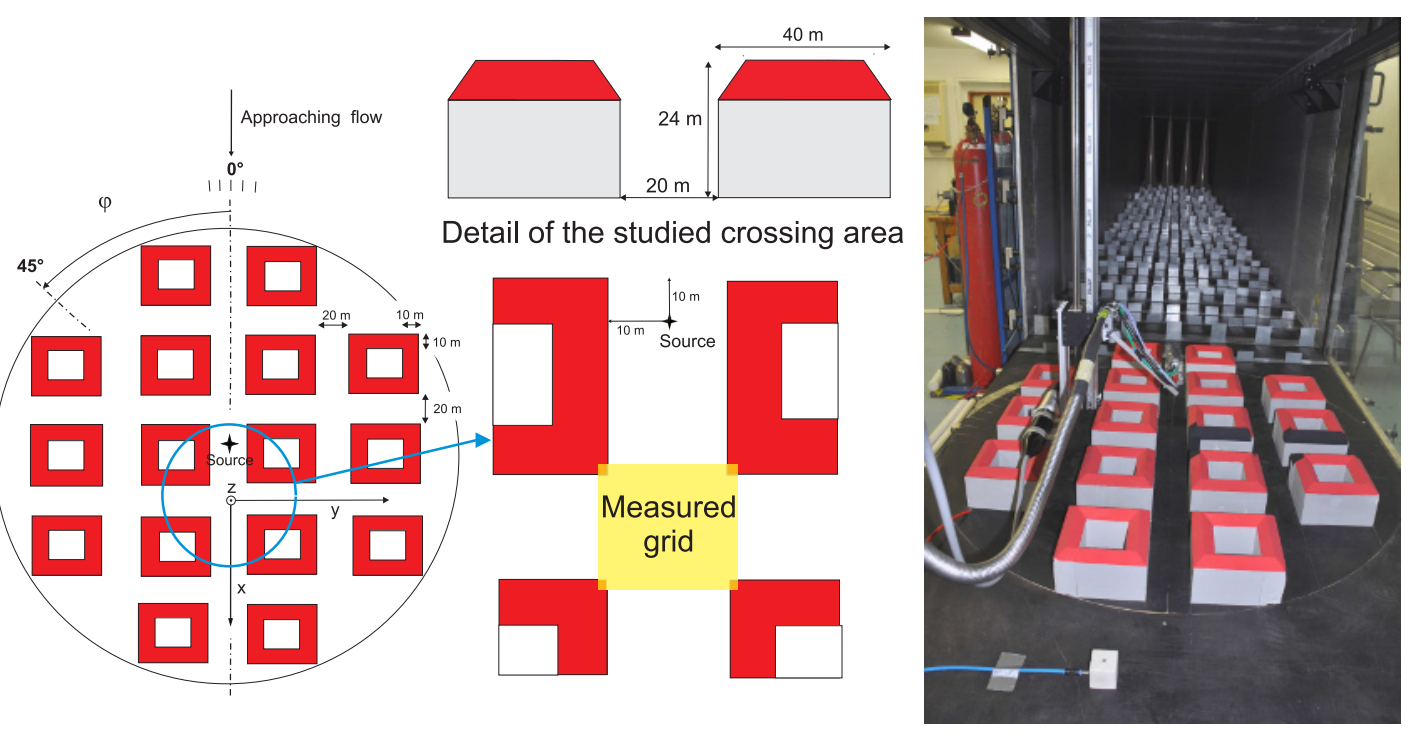

Fig. 2. Scheme of the idealised symmetric urban area model (left), the studied X-shaped intersection (middle) and the photograph of the model placed in the wind tunnel (right).

\subsection{Urban area model}

The model of idealised symmetric urban area with apartment houses was designed according to the common Central European inner-city area. Regular blocks of apartment houses with pitched roofs formed a perpendicular arrangement of the street canyons and X-shaped intersections, see figure 2 .

The model was scaled down to 1:200. The model buildings were formed by the body of height $100 \mathrm{~mm}$ and width $50 \mathrm{~mm}$ with pitched roof of height $20 \mathrm{~mm}$. We set up the characteristic building height $H=120 \mathrm{~mm}$ (24 m in full scale) as the height of building body with the roof.

The width of street canyons was $L=100 \mathrm{~mm}$. The aspect ratio of the street canyons given by the building height $H$ and the street width $S$ was $H / L=1.2$.

A point tracer gas source simulating a "pollution hot spot" (the place with higher emission of traffic pollution situated near a junction) was placed at the bottom of the street canyon in front of the studied intersection, see the scheme in figure 2.

\subsection{Measurement techniques}

The flow characteristics were measured by a two-dimensional optical fibre Laser Doppler Anemometry based on DANTEC BSA F-60 burst processor (LDA). Tracing particles (glycerine droplets with approximately $1 \mu \mathrm{m}$ diameter) were produced by a commercial haze generator placed at the beginning of the tunnel generating section, in front of turbulent generators. We got the air flow in the test section equally filled by seeding particles after running the haze generator inside the tunnel for several minutes. Data rate reached about $100 \mathrm{~Hz}$ at the bottom levels of street canyons $z \lesssim 0.5 \mathrm{H}$ and up to $1000 \mathrm{~Hz}$ at the roof top level $z \approx H$. The time of recording was $180 \mathrm{~s}$ in all the cases.

Point concentration measurements of tracer gas were realised by Fast-response Flame Ionisation Detector HFR400 Atmospheric Fast FID (FFID) made by Cambustion Ltd. The detector was set to acquire data at a data rate of
$1 \mathrm{KHz}$. The sampling time was $180 \mathrm{~s}$ in all of the cases. We used ethane as the tracer gas simulating passive pollutants. Ethane is passive and non-reactive gas with its own density $\rho_{\text {Ethane }}=1.24 \mathrm{~kg} \mathrm{~m}^{-3}$ close to density of the air $\rho_{\text {Air }}=1.28$ $\mathrm{kg} \mathrm{m}^{-3}$.

Simultaneous vertical velocity and concentration measurement at the roof top level above the intersection was realised using LDA and FFID. LDA and FFID probes were mounted on the traverse system in a way that the measuring volume of the LDA was close to the intake to the FFID sampling tube. The sampling tube intake was placed $1.5 \mathrm{~mm}$ above, $1 \mathrm{~mm}$ behind and $1 \mathrm{~mm}$ beside the centre of the LDA measuring volume, see figure 3.

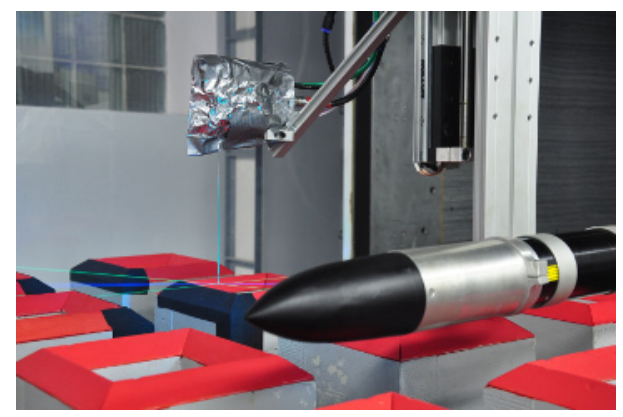

Fig. 3. The configuration of the FFID (left) and LDA (right) probes mounted on the traverse system in the wind tunnel.

As expected, the presence of the seeding particles in the air during simultaneous LDA and FFID measurement influenced FFID output signal. We got isolated spikes in the recorded concentration signal probably due to suction of combustible aerosol particles into the FFID probe, see $[11,12]$. We got similar count of spikes in time series obtained from measurements in clean air and in air contained seeding particles in most cases unlike these published results. We neglected the influence of spikes on the results because the frequency of isolated spikes was about $0.006 \%$ of used sampling data rate. 
The second influence of seeding particles on the measured concentration data was an almost constant shift of recorded concentration values caused obviously by sucking seeding particles by FFID probe. This shift reached about $0.5 \%$ of the FFID measuring range. The shift was corrected by the calibration sequence.

\subsection{Boundary layer characteristics}

Fully turbulent boundary layer was developed by spires and roughness elements placed it the tunnel. The characteristics of the boundary layer above the urban area model were measured with a two-dimensional LDA system in four vertical profiles placed above, upstream and downstream from the studied intersection, see figure 4.

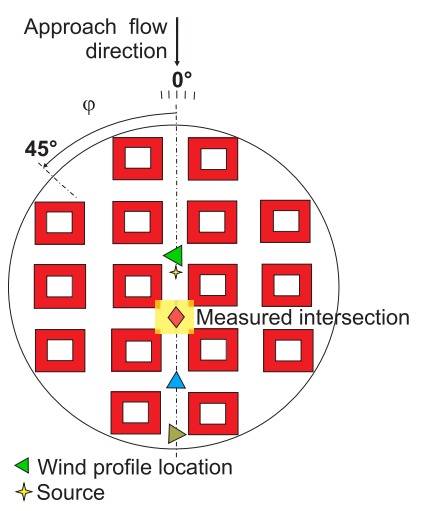

Fig. 4. Wind profile measurement locations.

The vertical profile of mean longitudinal velocity is depicted in figure 5a, the momentum flux profile can be found in figure $5 \mathrm{~b}$. The vertical profiles of longitudinal and vertical turbulent intensity are plotted in figures $5 \mathrm{c}$ and $5 \mathrm{~d}$. The high above the surface is expressed in full scale.

Vertical profiles of measured turbulent approach flow characteristics were fitted by the logarithmic and the power law. Mean roughness length $z_{0}$, displacement $d_{0}$ and friction velocity $u_{*}$ (alias square-root of constant Rey-nolds stress within the inertial sublayer) were obtained from the $\log$ wind profile fitting. Power exponent $\alpha$ was obtained from the power wind profile fitting. The parameters are listed in table 1 . Measured parameters corresponded to a

Table 1. Parameters of modelled boundary layer above the measured area (in full scale).

\begin{tabular}{llll}
\hline$z_{0}(m)$ & $d_{0}(m)$ & $\alpha(-)$ & $u_{*} / U_{2 H}(-)$ \\
\hline 0.83 & 13.40 & 0.24 & 0.096 \\
\hline
\end{tabular}

neutrally stratified boundary layer flow above a densely built-up area without much obstacle height variation. We used boundary layer classification according [13].

To verify requirements for the Townsend hypothesis [14] the critical Reynolds building number $R e_{B}$ was found. For our experiment, the modified Reynolds building number was given by

$$
R e_{B}=\frac{U_{2 H} H}{v},
$$

where $U_{2 H}$ is reference longitudinal velocity measured at a height of $z=2 H$ and $v$ is kinematic viscosity. This criterion is used for the flow within street canyons to be independent of viscous effects $[15,16]$. The experiment was carried out by $R e_{B} \approx 21000$ that lies on the lower edge of determined interval for valid Townsend hypothesis. Free stream velocity was approximately $4 \mathrm{~m} \mathrm{~s}^{-1}$.

\section{Results}

\subsection{Turbulent scalar flux fields}

The vertical and longitudinal velocity with concentration of tracer gas were simultaneously measured in a horizontal plane at the roof-top level $z=H$ above the studied intersection. Results were obtained for five approach flow angles $\varphi=0^{\circ}, 5^{\circ}, 15^{\circ}, 30^{\circ}$ and $45^{\circ}$.

The used Matlab post-processing script for synchronising simultaneously acquired vertical velocity and concentration data using the maximum of correlation between both signals. The synchronised time series were shifted by an average of $15 \mathrm{~ms}$. This shift expressed the delay between a suck of the sample into the intake of the FFID probe tube and the moment of the sample analysing in the probe. The value of the shift agrees with very similar experimental set up published by [12].

The dimensionless vertical turbulent scalar fluxes were computed from synchronised vertical velocity and concentration signals using eddy-correlation method $[17,18]$ using

$$
\left\langle c^{* \prime} w^{\prime}\right\rangle \frac{1}{U_{2 H}},
$$

where \langle\rangle is the time average, $c^{* \prime}$ and $w^{\prime}$ indicate fluctuations of dimensionless concentration and vertical velocity, respectively (see similar approach in [5]). These computed fluxes express a rate of emissions spreading through a unit area by turbulent transport. The positive sign means the flux outwards and the negative sign means the flux inwards the street intersection.

Values of determined vertical turbulent fluxes for the four approach flow directions are plotted in figure 7 . We measured relatively flat turbulent flux field with small and positive values by angle $0^{\circ}$, see figure $7 \mathrm{a}$. In case $15^{\circ}$ there are significantly positive values on the upwind side of the area, see figure $7 \mathrm{c}$. This phenomenon became stronger by angle $45^{\circ}$, see figure $7 \mathrm{~d}$. We estimated a significant turbulent transport of pollution near the leeward side of the buildings, see the upper part of figures $7 \mathrm{a}$ and $7 \mathrm{~b}$.

\subsection{Quadrant analysis}

The quadrant analysis was applied to the synchronised velocity and concentration fluctuation time series. We used usual nomenclature published in [19]:

$$
\begin{aligned}
& 1^{\text {st }} \text { quadrant "outward interaction" }\left(x^{\prime}>0, w^{\prime}>0\right), \\
& 2^{\text {st }} \text { quadrant "sweep" }\left(x^{\prime}>0, w^{\prime}<0\right), \\
& 3^{\text {st }} \text { quadrant "inward interaction" }\left(x^{\prime}<0, w^{\prime}<0\right), \\
& 4^{\text {st }} \text { quadrant "ejection" }\left(x^{\prime}<0, w^{\prime}>0\right),
\end{aligned}
$$




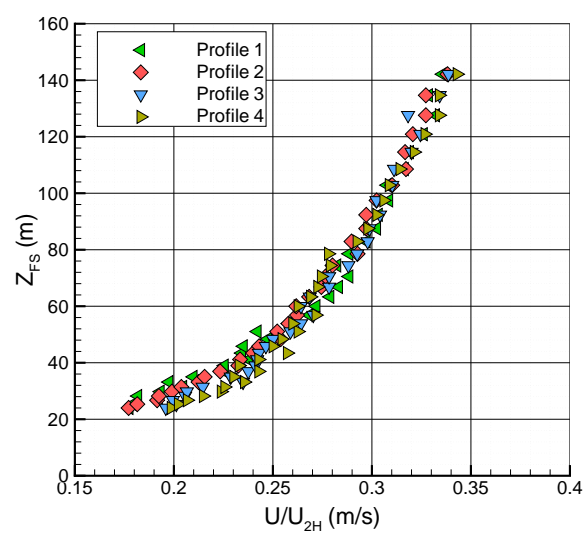

(a) The vertical profiles of mean longitudinal velocity.

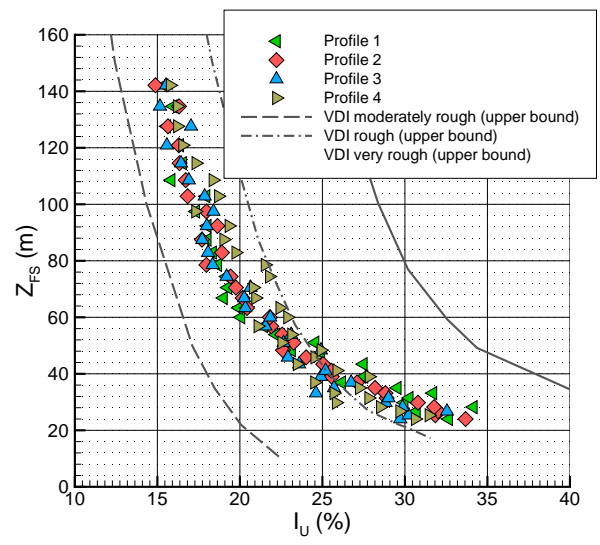

(c) The vertical profiles of longitudinal turbulent intensity.

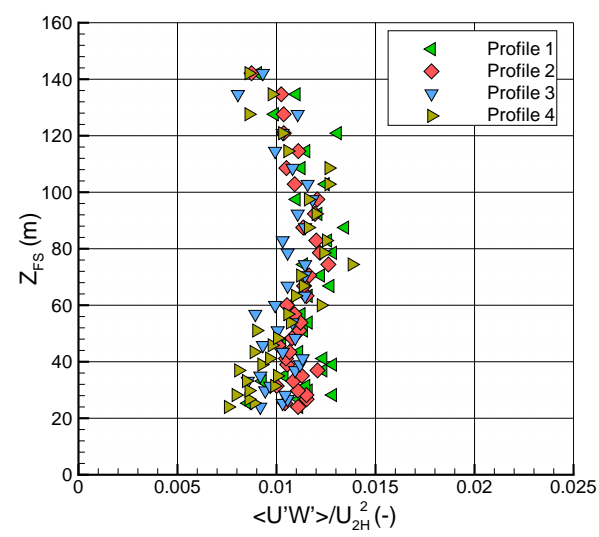

(b) The vertical profiles of mean momentum flux.

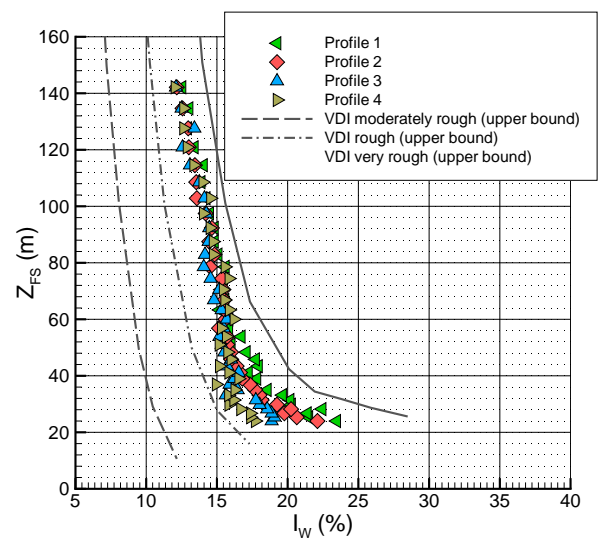

(d) The vertical profiles of vertical turbulent intensity.

Fig. 5. Boundary layer characteristics above the urban area model.

where $x^{\prime}$ represents dimensionless concentration fluctuation $c^{* \prime}$. These definitions are illustrated in figure 6 bellow. The threshold time and value was used to identify individual events in fluctuation signals. The threshold time was set to $2 \mathrm{~ms}$ as a duration of two consecutive time steps in measured signal. The threshold value was used 0.0005 (-) as a minimum value that can be resolved from an electric noise in the signals.

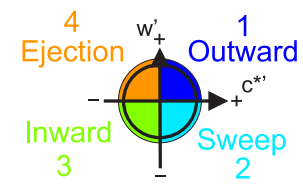

Fig. 6. The scheme of event definitions used in quadrant analysis of turbulent pollution flux.

The particular contribution from $i^{t h}$ quadrant to the total turbulent pollution flux $\left\langle c^{* \prime} w^{\prime}\right\rangle / U_{2 H}$ is given by

$$
S_{i}=\frac{\left\langle c^{*} w^{\prime}\right\rangle_{i} N_{i}}{N_{\text {total }}}
$$

where $N_{i}$ is the number of events in the $i^{\text {th }}$ quadrant and $N_{\text {total }}$ is number of all measured events.
The relative contribution of the prevailing event to the total scalar flux was computed as

$$
\frac{S_{\max }}{\sum S_{i}} 100 \%
$$

where $S_{\max }$ is the particular contribution from the dominant event. These contributions of the prevailing events are plotted in figure 8 for four approach flow directions. As you see in figures $8 \mathrm{a}, 8 \mathrm{~b}$ and $8 \mathrm{c}$, outward interactions dominated in the area for smaller approach wind directions. It means, that particles of air with a positive fluctuation of the vertical velocity and concentration were transported upwards from the intersection. There is a large area with domination of inward interaction for approach flow angle $\varphi=45^{\circ}$, see figure $8 \mathrm{~d}$. The positive vertical turbulent flux is formed mostly by downwards moving particles of fresh air in this area.

The mean dimensionless duration was computed for the dominant events as

$$
\left\langle t_{i}\right\rangle_{S_{\max }} \frac{U_{2 H}}{H}
$$

where $t_{j}$ is the measured duration of the dominant event. Values of mean durations are depicted in figure 9 for four approach flow angles. 
The mean dimensionless repetition frequency was computed for dominant events using

$$
\left\langle\frac{1}{\tau_{j}}\right\rangle_{S_{\max }} \frac{H}{U_{2 H}},
$$

where $\tau_{j}$ is the measured duration between two dominant events. Computed repetition frequencies are plotted in figure 10 for the four approach flow directions.

We can compare figures 7, 9 and 10 now. It is obvious that the outward interactions with low repetition frequencies and relatively long durations dominated in a low and positive vertical turbulent transport for lower approach flow angles $0^{\circ}$ and $5^{\circ}$; compare figures $9 \mathrm{a}$ and $10 \mathrm{a}$, figures $9 \mathrm{~b}$ and $10 \mathrm{~b}$. The repetition frequency increased and duration slightly decrease in case of angle $15^{\circ}$, compare figures $9 \mathrm{c}$ and 10c. This can be observed by angle $30^{\circ}$, as well (not shown). The inward interactions with high frequencies and long durations dominated in the intensive positive turbulent flux in the last situation by approach flow angle $45^{\circ}$, see figures $9 \mathrm{~d}$ and $10 \mathrm{~d}$.

Measured values of the mean dimensionless duration of dominant events between $0.20-0.45$ correspond to durations around 1.6-3.6 $\mathrm{s}$ in a real symmetric urban area with $H=24 \mathrm{~m}$ and $U_{2 H}=3 \mathrm{~m} \mathrm{~s}^{-1}$. In case of repetition frequencies, measured values $0.5-1.2$ correspond to $0.06-0.15 \mathrm{~Hz}$. It means that periods of events reach values around $6.5-16.0 \mathrm{~s}$.

The duration percentage of the dominant events was computed as the last quantity by

$$
\frac{\Sigma t_{j S_{\max }}}{\Sigma t_{j}} 100 \%
$$

where $t_{j S_{\max }}$ is the duration of the dominant event and $t_{i}$ is duration of every detected event. The duration percentage is shown in figure 11 for the four approach flow directions. The dominate outward interactions influenced the vertical pollution turbulent flux for a relatively short time in lower approach flow angles $0^{\circ}$ and $5^{\circ}$, see figures $11 \mathrm{a}$ and $11 \mathrm{~b}$. The duration percentage of outward interaction obviously increase with increasing angle, see figure $11 \mathrm{c}$. The dominant inward interaction were detected in up to $40 \%$ of measured period in case of angle $45^{\circ}$, see figure $11 \mathrm{~d}$.

\section{Conclusions}

Vertical turbulent pollution fluxes were measured in a horizontal plane above the modeled X-shaped street intersection in an idealized symmetrical urban area for five wind directions. An experimental set-up for simultaneous measurement of the flow velocity and the tracer gas concentration was designed and assembled, based on Fast-response Flame Ionisation Detector and Laser Doppler Anemometer.

The influence of the approach flow direction on the vertical turbulent pollution fluxes were determined. The increasing vertical turbulent pollution flux was observed with diverging approach flow direction from the street with pollution source.

The quadrant analysis was applied to the vertical turbulent pollution fluxes data. We determined that the vertical turbulent pollution flux is caused by transport of polluted air particles upward from the intersection (outward interactions) in case of approach flow almost parallel to the street canyon with the pollution source. The turbulent pollution flux reach low magnitude in these cases. Outward interactions reached low repetition frequencies and relatively long durations. In general, the outward interactions influenced the vertical pollution turbulent flux for a relatively short time compared with the total duration of all detected events.

Transport of fresh air downward into the street intersection (inward interaction) dominated in the vertical turbulent flux for diverging approach flow from the street with the pollution source. These dominate events reached high repetition frequencies and long durations. The outward interactions were present in almost half of the total duration of all detected events.

\section{Acknowledgement}

The authors kindly thank the Ministry of Education, Sports and Youth of the Czech Republic (project AVOZ-20760514), Charles University in Prague (projekt GAUK No. 535412) and the Czech Science Foundation GACR (project GAP101/12/1554) for their financial support.

\section{References}

1. J. Fenger, Atmos. Environ. 33, 4877 (1999)

2. G. Hoek, B. Brunekreef, A. Verhoeff, J. van Wijnen, P. Fischer, Journal of the Air and Waste Management Association 50, 1380 (2000)

3. L. Kukačka, R. Kellnerová, K. Jurčáková, Z. Jaňour, Air pollution modelling and its application XXI (Springer, Dordrecht, 2012), chap. Analysis of scalar fluxes and flow within modelled intersection depending on the approach flow direction, pp. 113-118

4. M. Carpentieri, A. Robins, Boundary-Layer Meteorol 135, 229 (2010)

5. M. Carpentieri, P. Hayden, A.G. Robins, Atmos. Environ. 46, 669 (2012)

6. L. Soulhac, V. Garbero, P. Salizzoni, P. Mejean, R. Perkins, Atmos. Env. 43, 2981 (2009)

7. R. Kellnerová, Acta Technica 54, 401 (2009)

8. B. Feddersen, Ph.D. thesis, ETH Zürich (2004)

9. G. Katul, G. Kuhn, J. Schildge, C. Hsieh, Bound.-Layer Meteor. 83, 1 (1997)

10. M. Raupach, J. Fluid Mech. 108, 363 (1981)

11. D. Hall, M. Emmont, Experiments in Fluids 10, 237 (2006)

12. D. Contini, P. Hayden, A. Robins, Atmospheric Environment 40, 7842 (2006)

13. V.D. Ingenieure, ed., Physical modelling of flow and dispersion processes in the atmospheric boundary layer - application of wind tunnels (VDI Verein Deutcher Ingenieure, Düsseldorf, 2000)

14. A. Townsend, A Structure of Turbulent Shear Flow (Cambridge University Press, New York, 1976)

15. R.N. Meroney, M. Pavageau, S. Rafailidis, M. Schatzmann, J. Wind. Eng. Ind. Aerodyn. 62, 37 (1996)

16. M. Pavageau, M. Schatzmann, Atmos. Environ. 33, 3961 (1999)

17. S. Arya, Air pollution meteorology and dispersion (Oxford University Press, New York, 1999)

18. R. Stull, An Introdution to Boundary Layer Meteorology (Kluwer Aademic Publishers, Dordrecht, 1988)

19. W.W. Willmath, S.S. Lu, Adv. Geophys. 18A, 287 (1974) 


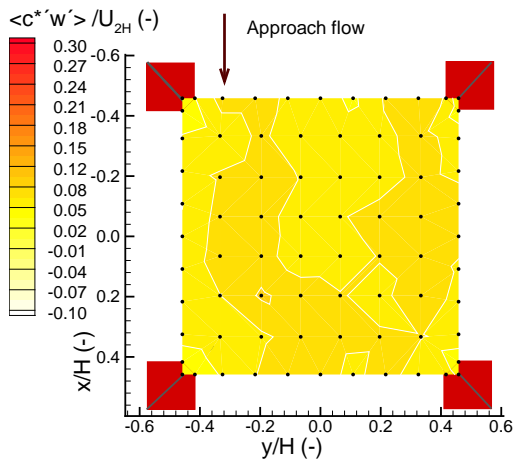

(a) Approach flow $\varphi=0^{\circ}$.

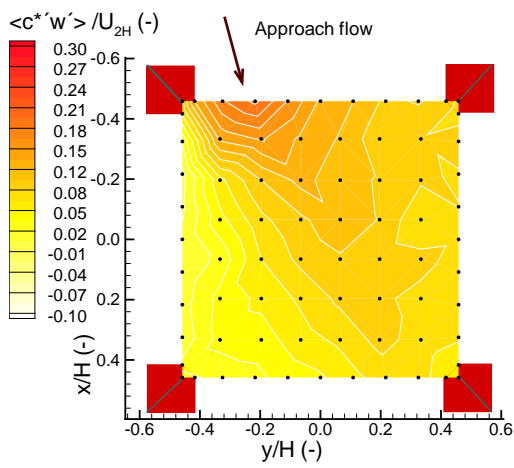

(c) Approach flow $\varphi=15^{\circ}$.

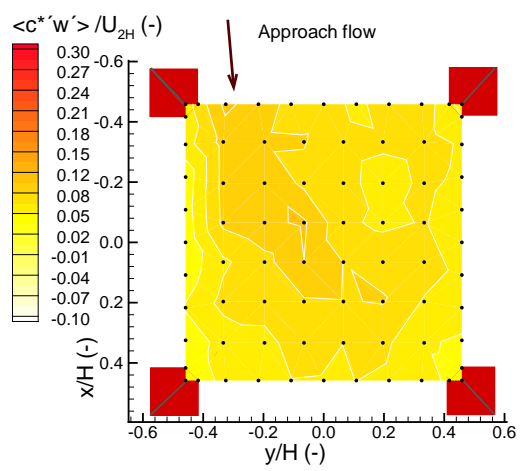

(b) Approach flow $\varphi=5^{\circ}$.

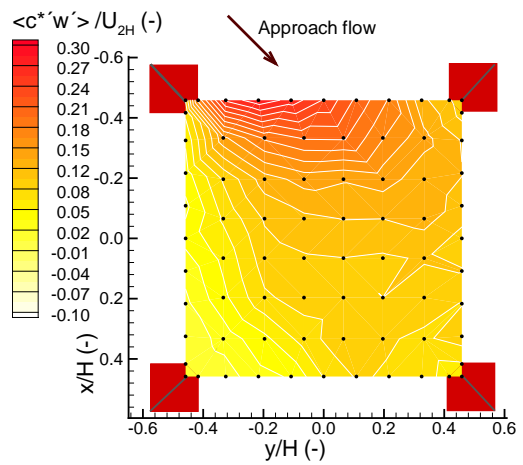

(d) Approach flow $\varphi=45^{\circ}$.

Fig. 7. Vertical dimensionless turbulent scalar flux $\left\langle c^{* \prime} w^{\prime}\right\rangle / U_{2 H}$ for four angles of the approach flow direction.

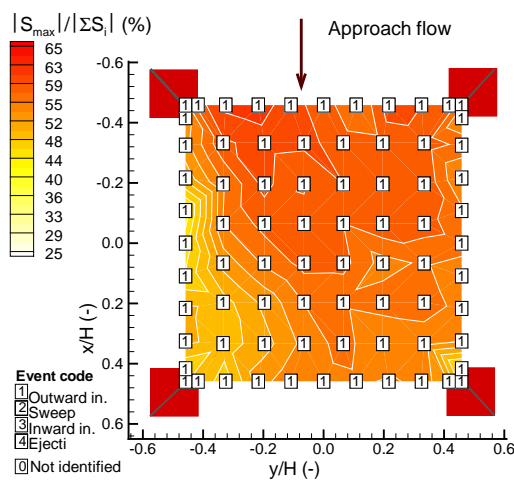

(a) Approach flow $\varphi=0^{\circ}$.

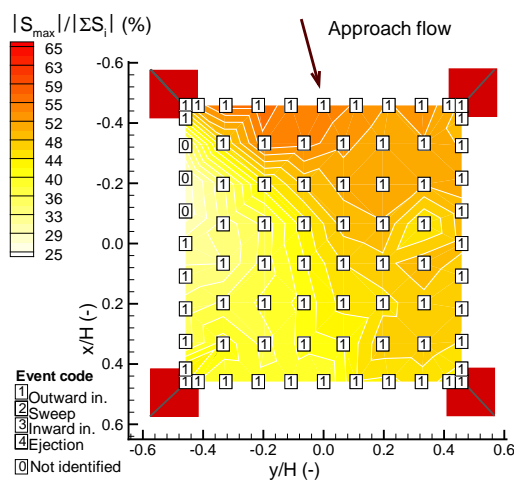

(c) Approach flow $\varphi=15^{\circ}$.

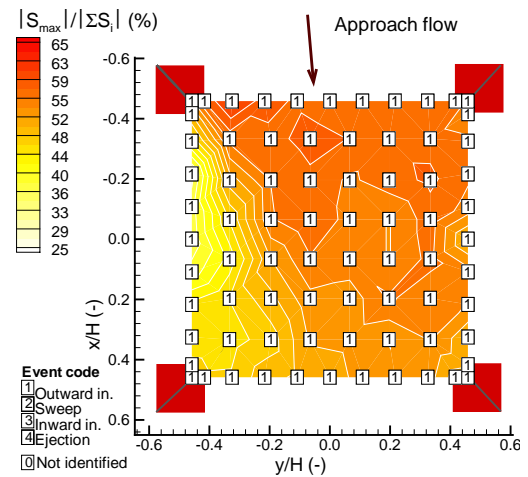

(b) Approach flow $\varphi=5^{\circ}$.

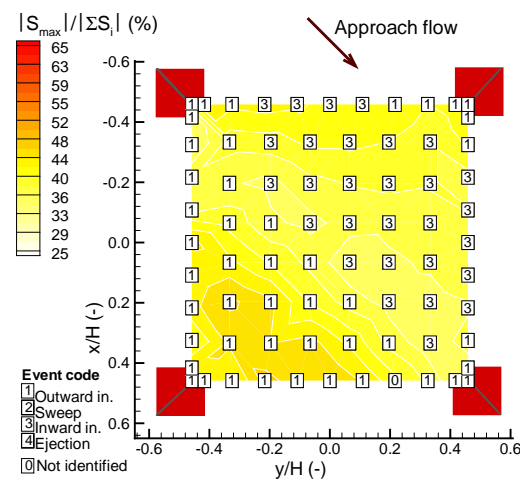

(d) Approach flow $\varphi=45^{\circ}$.

Fig. 8. The relative contribution of the prevailing event to the total scalar flux $S_{\max } / \sum S_{i} 100 \%$. 


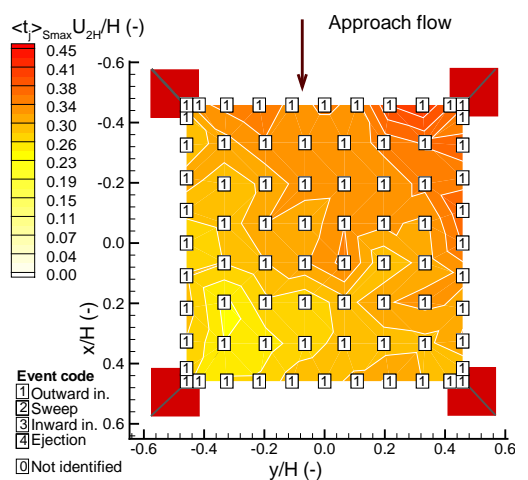

(a) Approach flow $\varphi=0^{\circ}$.

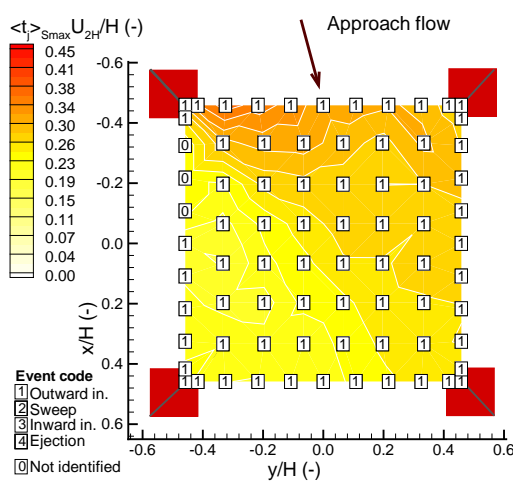

(c) Approach flow $\varphi=15^{\circ}$.

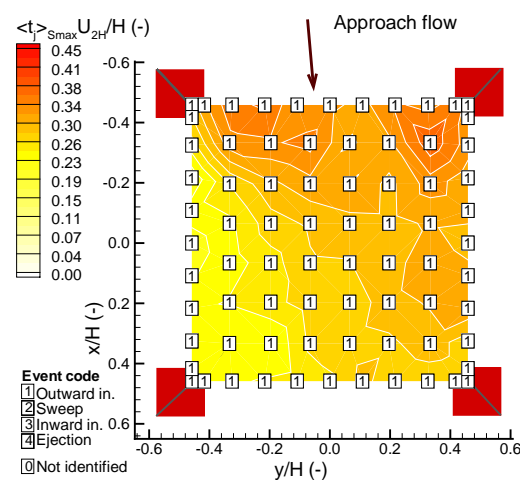

(b) Approach flow $\varphi=5^{\circ}$.

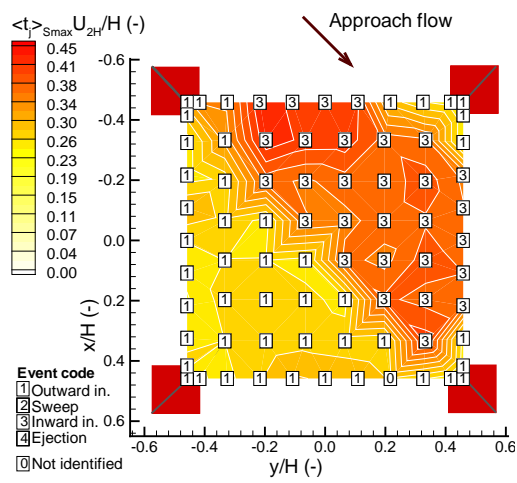

(d) Approach flow $\varphi=45^{\circ}$.

Fig. 9. The mean dimensionless duration of the dominant events $\left\langle t_{j}\right\rangle_{S_{\max }} U_{2 H} / H$.

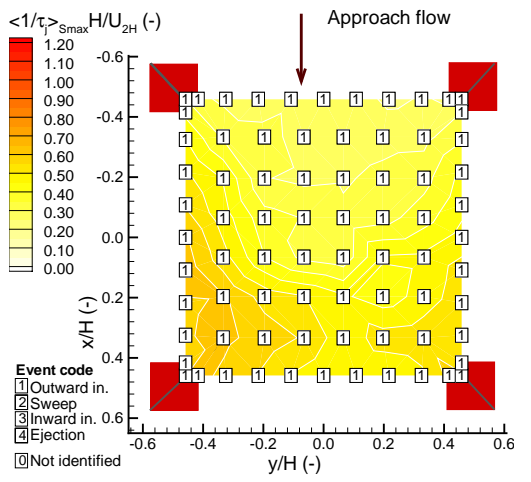

(a) Approach flow $\varphi=0^{\circ}$.

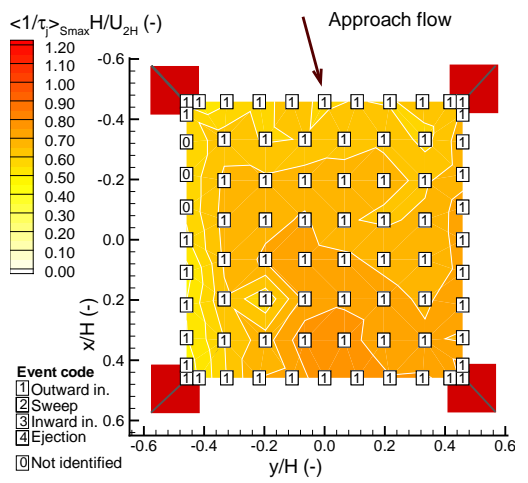

(c) Approach flow $\varphi=15^{\circ}$.

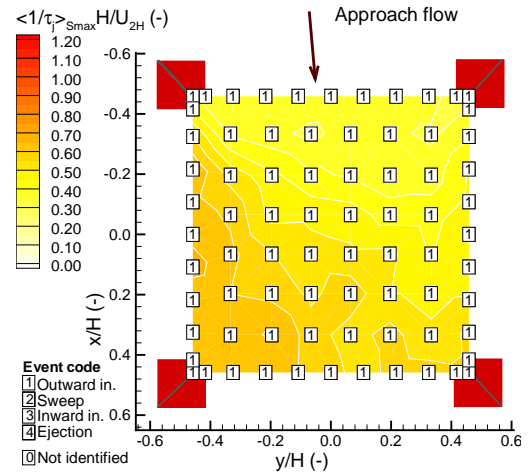

(b) Approach flow $\varphi=5^{\circ}$.

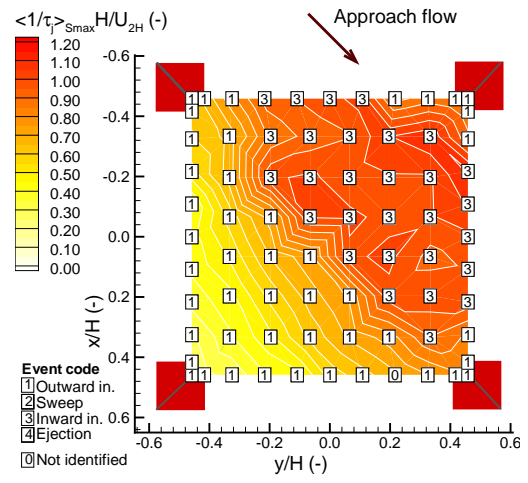

(d) Approach flow $\varphi=45^{\circ}$.

Fig. 10. The mean dimensionless repetition frequency of the dominant events $\left\langle 1 / \tau_{j}\right\rangle_{S_{\max }} H / U_{2 H}$. 


\section{EPJ Web of Conferences}

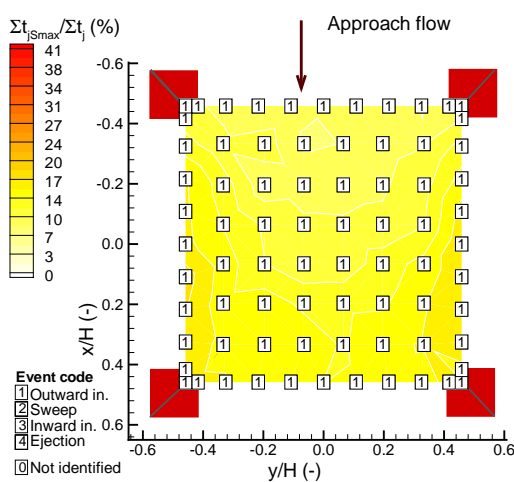

(a) Approach flow $\varphi=0^{\circ}$.

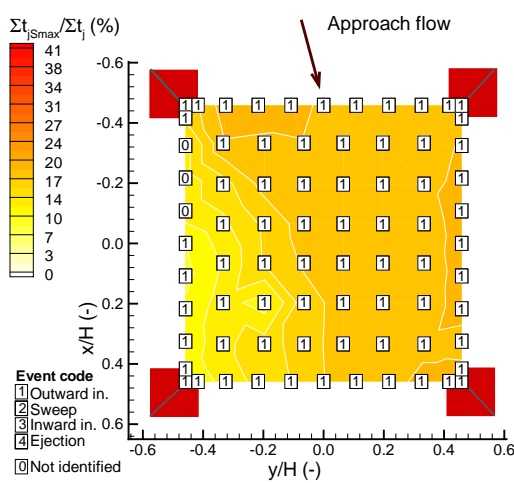

(c) Approach flow $\varphi=15^{\circ}$.

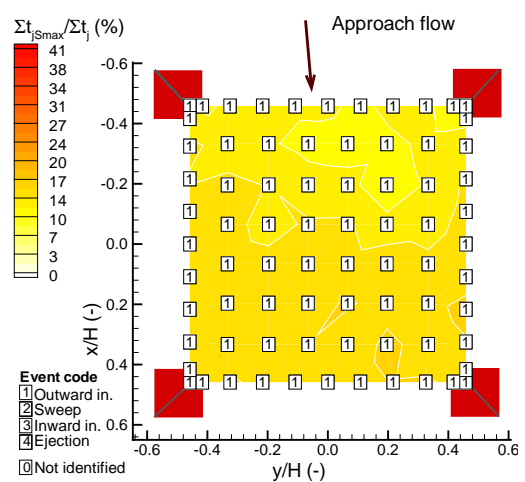

(b) Approach flow $\varphi=5^{\circ}$.

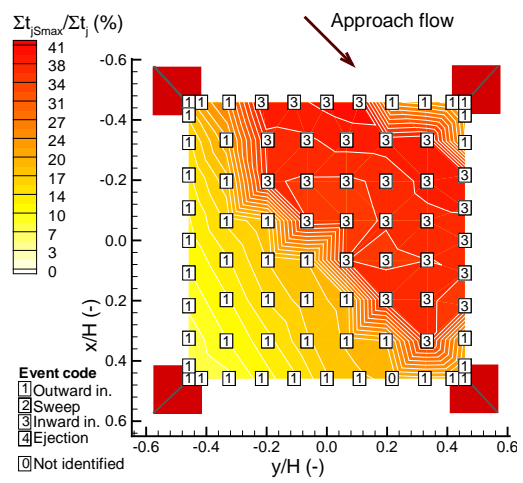

(d) Approach flow $\varphi=45^{\circ}$.

Fig. 11. The duration percentage of the dominant events $\Sigma t_{j S_{\max }} / \Sigma t_{j} 100 \%$. 